\section{A case report of inadvertent intranasal submucosal injection of concentrated epinephrine with no long-term sequelae}

\author{
Karlyn Powell
}

Department of Anesthesiology, Baylor College of Medicine Baylor St. Luke's Medical Center, Houston, Texas, USA

\section{Dear Editor,}

Despite awareness of medication errors involving epinephrine and its different concentrations, drug errors continue to occur. Reported cases with the mistaken use of concentrated epinephrine had poor outcomes. This case report describes a favorable outcome despite the inadvertent use of concentrated epinephrine during a transphenoidal tumor resection and offers possible explanations for this outcome and methods to avoid similar incidents in the future.

The application of epinephrine is popular to optimize conditions for endoscopic sinus surgery. Surgeons may tropicalize with a vasoconstrictor or infiltrate with a local anesthetic containing epinephrine or both. For topicalisation pledgets soaked with phenylephrine, oxymetazoline or concentrated epinephrine are applied to the nasal mucosa to aid with hemostasis. Lidocaine in combination with a less concentrated epinephrine is injected to blunt the hemodynamic response with the role of epinephrine to increase the duration of action of the local anesthetic, reduce local anesthetic toxicity, and decrease bleeding.

Epinephrine's a-adrenergic properties cause the desired local vasoconstriction but its $\beta$-adrenergic effects and variable systemic absorption can cause complications. a mediated vasoconstriction increases vascular resistance resulting in systemic and pulmonary hypertension. $\beta$ stimulation can cause increased heart rate and cardiac contractility which increases cardiac output and myocardial demand and lead to arrhythmias. Unpredictable systemic absorption can cause toxic levels causing hypertensive crisis and fatal arrhythmias. Additionally, left ventricular failure and pulmonary edema can occur.

Different concentrations of epinephrine are used for topical application and infiltration of local anesthetic. Errors in medication concentration can occur as the different concentrations can be used in the same procedure. Reported cases in which 1:1000 concentrations were mistakenly used during infiltration instead of topical application tended to have poor aftermaths. The Canada Safety Bulletin highlights two similar ENT cases in which patients were injected with 1:1000 epinephrine intended for topical use. In one incident the patient recovered without permanent harm but in the other incident the patient experienced an arrhythmia leading to cardiac arrest and death [1]. Orlandi [2] describes a patient developing transient hypertension and pulmonary edema after erroneous preparation and injection of 1:1000 epinephrine. Chelliah [3] details a hypertensive crisis causing myocardial ischemia which occurred during an injection of $1.5 \mathrm{~mL}$ of lidocaine $1 \%$ with 1:100,000 epinephrine following topicalisation with 1:1000 epinephrine packing. The contents of the syringe were not analyzed but there was a possibility that a stronger concentration of epinephrine was injected.

\section{CASE DESCRIPTION}

A 30 -year-old $69 \mathrm{~kg}$ female with a past medical history notable only for hyperprolactinemia presented for an endoscopic transphenoidal pituitary tumor resection to be performed jointly by ENT and neurosurgery. Preoperative imaging showed a $7 \mathrm{~mm}$ right internal carotid aneurysm in addition to the $18 \times 22 \times 20 \mathrm{~mm}$ pituitary macroadenoma.

After patient history and consent were obtained, the patient was sedated and taken to the operating room where she was placed under a general anesthesia with an endotracheal tube. An additional peripheral IV and a radial arterial line were placed prior to the bed being rotated $180^{\circ}$ and the arms tucked.

Nineteen minutes after intubation the ENT resident informed the anesthesiologist that he was about to localize the nasal mucosa with lidocaine $1 \%$ with epinephrine. Within thirty seconds of his announcement the blood pressure and heart rate dramatically increased to $220 / 160 \mathrm{~mm}$ $\mathrm{Hg}$ and 150 per min, respectively. An additional bolus of propofol was given and the volatile gas concentration was increased as it was initially assumed that the patient was reacting to the epinephrine in the injection of local anesthestic. As the blood pressure and heart rate remained high for a few minutes despite interventions, the patient's arm was untucked to assess if the arterial line tracing had been compromised. The arterial line appeared to be functioning properly; however, it was noted that the patient's hand was cold and blanched.

It was determined that the ENT resident had injected $9 \mathrm{mg}$ of topical 1:1000 epinephrine erroneously thinking it was a syringe of lidocaine $1 \%$ with 1:100,000 epinephrine despite the correct labeling of the syringe. An intraoperative CT was performed which ruled out an intracranial bleed from her aneurysm. However, an EKG showed ST depressions. A nitroglycerin infusion was considered but the high volatile concentration immediately after the event and propofol boluses adequately decreased the blood pressure. After ninety minutes the blood pressure gradually normalized, and ST depressions lessened, the decision was made to extubate the patient. Postoperatively the patient was able to follow commands, answer questions, and move all extremities and she denied chest pain or headache. On arrival to the recovery room her heart rate was in the high 
80 s with a small ST depression still present and blood pressure in the $90 \mathrm{~s} / 50 \mathrm{~s}$.

The patient was transferred from the recovery room to the cardiac intensive care unit for further monitoring. Her initial troponin was $0.95 \mathrm{ng} \mathrm{mL}^{-1}$ which decreased over the next few days as did her ST depressions on EKG. A transthoracic ECHO was unremarkable. The patient continued to deny chest pain and headache postoperatively. She returned to the OR four days later after cardiology clearance and underwent the procedure without complication. The nasal mucosa was noted to appear healthy at this time. Of note, surgery used pledgets soaked with phenylephrine in lieu of epinephrine during the procedure. The patient was discharged home the following day.

\section{DISCUSSION}

The fortunate outcome for this patient may be attributed to an array of factors. Foremost, the patient's young age and lack of comorbidities were crucial specifically her lack of cardiac or vascular disease. Secondly, preprocedure placement of an arterial line enabled early detection of the event as well as guided management. After the initial spike in blood pressure to $220 / 160 \mathrm{~mm} \mathrm{Hg}$ and heart rate to 150 per min, respectively, the blood pressure was decreased to $120 / 100 \mathrm{~mm} \mathrm{Hg}$ within five minutes and the heart rate was decreased to the $120 \mathrm{~s}$ within twenty minutes. Furthermore, a high volatile concentration and propofol boluses created adequate vasodilation to counter the epinephrine's a mediated increases in vascular resistance.

Additionally, specific medications known to have deleterious effects with epinephrine were not given. $\beta$ blockers and calcium channel blockers were not given as these medications can blunt the compensatory mechanisms of increased heart rate and myocardial contractility which prevent overload of the pulmonary vasculature and maintain cardiac output in the face of a significantly increased afterload. Schwalm et al. [4] describes a case in which patient who underwent an ENT procured with epinephrine (1:1000) packing and injection with $4 \mathrm{~mL}$ of lidocaine $1 \%$ with epinephrine $(1: 200,000)$ who became hypertensive and tachycardic postoperatively presumably due to epinephrine absorption. The patient was given metoprolol and subsequently developed pulmonary edema, PEA, and cardiogenic shock. The case reflects a series of ENT cases by described Kalyanaraman [5], in which patients who were given topical phenylephrine or submucosally injected epinephrine developed pulmonary edema after being given $\beta$-blockers or calcium channel blockers. Also, nitrous oxide was not used which could have contributed to an increased vascular resistance and increased pulmonary artery pressure.
Lastly, as epinephrine absorption is variable it is likely that the patient did not undergo the full effects of $9 \mathrm{mg}$ of concentrated epinephrine. The mucous membranes of the nasal cavity are highly vascular yet local submucosal vasoconstriction by the high concentration may have prevented further systemic absorption of the medication. Further, it is possible that the systemically absorbed epinephrine did not remain in the blood significantly longer than less concentrated versions of the medication. Matousek and colleagues [6] monitored blood epinephrine concentrations during a study during which they discovered $6 \mathrm{~mL}$ of an incorrect higher concentration had been injected into the nasal mucosa of two patients. After 10 minutes, the patients who had received 1:10,000 had similar venous epinephrine concentrations as the 14 patients who had received 1:100,000.

Subsequent meetings involving anesthesiology, ENT, neurosurgery, and OR nursing determined a systemic cause to this life-threatening incident. Typically, in these cases, the circulating nurse prepares a medicine bowl containing $30 \mathrm{~mL}$ topical 1:1000 epinephrine for soaking pledgets and draws the syringe containing $10 \mathrm{~mL}$ lidocaine 1\% with 1:100,000 epinephrine. These items are placed on a back table for the surgeon's use prior to prepping and draping.

In this case, the topical epinephrine in pour bottles was backordered and the nurse drew the epinephrine from multiple $1 \mathrm{~mL}$ vials using a $10 \mathrm{~mL}$ syringe with a needle. The syringe was labeled as containing epinephrine 1:1000 and placed on the back table next to an empty medicine cup. The resident assumed that the syringe contained lidocaine with epinephrine for his use despite being clearly labeled as epinephrine.

Several changes were made to the OR practice based on this incident. Topical 1:1000 epinephrine is poured into Petri dishes and colored with a marker tip indicating that it is the solution for soaking pledgets. Epinephrine can remain on the field only in open containers instead of syringes. Surgeons no longer use lidocaine with epinephrine and there are no syringes in the field.

\section{ACKNOWLEDGEMENTS}

Declaration of conflicting interests: The author declared no potential conflicts of interests with respect to the research, authorship, and/or publication of this article. Funding: The author received no financial support for the research, authorship, and/or publication of this article. Statements: This paper has not been published in whole or in part in any other journal.

Written patient consent was obtained before publication of this report. 


\section{References:}

1. ISMP Canada Safety Bulletin. 2009; 9(2): 1-3.

2. Orlandi RR, Warrier $S$, Sato $S$, et al. Concentrated topical epinephrine is safe in endoscopic sinus surgery. Am J Rhinol Allergy. 2010; 24(2): 140-142, doi: 10.2500/ajra.2010.24.3454, indexed in Pubmed: 20338114

3. Chelliah YR, Manninen PH. Hazards of epinephrine in transsphenoidal pituitary surgery. J Neurosurg Anesthesiol. 2002; 14(1): 43-46, indexed in Pubmed: 11773822.

4. Schwalm JD, Hamstra J, Mulji A, et al. Cardiogenic shock following nasal septoplasty: a case report and review of the literature. Can J Anaesth. 2008; 55(6): 376-379, doi: 10.1007/BF03021494, indexed in Pubmed: 18566202

5. Kalyanaraman M, Carpenter RL, McGlew MJ, et al. Cardiopulmonary compromise after use of topical and submucosal alpha-agonists: possible added complication by the use of beta-blocker therapy.
Otolaryngol Head Neck Surg. 1997; 117(1): 56-61, doi: 10.1016/S019459989770207-9, indexed in Pubmed: 9230324.

6. Matoušek P, Komínek P, Garčic A. Errors associated with the concentration of epinephrine in endonasal surgery. Eur Arch Otorhinolaryngol. 2011;268(7): 1009-1011, doi: 10.1007/s00405-010-1435-4, indexed in Pubmed: 21328004.

\section{Corresponding author:}

Karlyn Powell, M.D.

Department of Anesthesiology

Baylor College of Medicine

One Baylor Plaza

MS: BCM120

Houston, Texas 77030, USA

e-mail:Karlyn.Powell2@bcm.edu
Anaesthesiology Intensive Therapy 2018 , vol. 50 , no 4,323 ISSN $1642-5758$ 0.5603/AIT.a2018.0035 www.ait.viamedica.pl

\section{Peri-anaesthetic cardiac arrest with administration of enalapril, spironolactone and $\beta$-blocker}

\section{Cedric Basquin, Catherine Simeon, Eric Dupont-Bierre, Marc Gentili}

\section{Centre Hospitalier Privé Saint-Grégoire, France}

Although cardiac arrest during anaesthesia is rare, it may be of grave concern for the patient. The mechanism is a multifactor event, including personal treatment. Antihypertensive and diuretic drugs can induce dyskalaemia and heart rhythm problems per se while others, such as $\beta$-blockers, can controversially generate pulseless electrical activity. We report the occurrence of unanticipated peri-anaesthetic cardiac arrest where the use of such drugs need to be debated.

A 77-year-old male (ASA 3, NYHA 2) was scheduled for ambulatory laparoscopic hernia repair. He had a medical history of myocardial ischaemia, hypertension and stable aortic valvulopathy, as well as renal insufficiency (glomerular filtration rate: $33 \mathrm{~mL} \mathrm{~min}^{-1}$ ). He was considered as stable and asymptomatic by his cardiologist. He had received daily acetylsalicylate acid $75 \mathrm{mg}$, bisoprolol $10 \mathrm{mg}$, lercandipine $10 \mathrm{mg}$, spironolactone $50 \mathrm{mg}$ and enalapril $20 \mathrm{mg}$. The coagulation parameters and blood group were checked. Laparoscopic surgery was planned under general anaesthesia (remifentanil and sevflurane). Routine monitoring included heart rate, ECG, noninvasive blood pressure and pulse oximetry, baseline parameters were normal. The surgical procedure lasted around 45 minutes: gas insufflation and exsufflation were uneventful. Suddenly, his heart rate dropped from 65 beats per min to asystole while $\mathrm{SpO}_{2}$ fell to $72 \%$ and capnometry to $20 \mathrm{mmHg}$ while noninvasive blood pressure (BP) became unmeasureable and peripheral pulses could not be palpated. Cardiopulmonary resuscita- tion was started immediately, including two repeated intravenous doses of $1 \mathrm{mg}$ epinephrine and $100 \%$ oxygen while sevoflurane was suppressed. Within 2 minutes, the patient responded with a heart rate of $160 \mathrm{bpm}, \mathrm{SpO}_{2} 92 \%$ and $\mathrm{BP}$ of $90 / 60 \mathrm{mmHg}$. The patient was admitted to PACU where the ECG became progressively sinusoidal at around 105 per min. Echocardiography (CB) eliminated a gaseous or pulmonary embolism by the absence of acute right ventricular heart failure. A moderate aortic stenosis was discovered with an aortic valve area of around $0.6 \mathrm{~cm}^{2}$, a pressure gradient at $25 \mathrm{~mm} \mathrm{Hg}$ and a left ventricular ejection fraction estimated at around $60 \%$. No aspect of myocardial infarction or severe pericarditis was observed. BNP — was at $770 \mathrm{ng} \mathrm{mL}^{-1}$, troponins around $30 \mathrm{\mu g} \mathrm{mL}^{-1}, \mathrm{pH}-7.14, \mathrm{pCO}_{2}-32 \mathrm{~mm}$ $\mathrm{Hg}, \mathrm{pO}_{2}-153 \mathrm{~mm} \mathrm{Hg}$, while electrolytes were in a normal range, apart from potassium at $6.29 \mathrm{mEq} \mathrm{L}^{-1}$. A chest X-ray showed no abnormality. The patient was admitted to the intensive care unit to undergo haemodialysis. He was extubated on day 1 with a $\mathrm{pH}$ of 7.44 and a potassium level of $4.39 \mathrm{mEq} \mathrm{L}^{-1}$. The patient was discharged on day 4 and gave his informed consent to publish this case. Spironolactone was substituted with furosemide.

Obviously, the role of hyperkalaemia has to be discussed. Most hyperkalaemia-induced cardiac arrests may be observed in patients with neuromuscular dystrophy, blood transfusion or iatrogenic infusion [1, 2]. A combination of spironolactone with an ACE inhibitor may cause severe hyperkalaemia [3]. Abbas et al. [4], using health insurance claims data and performing a nested case-control study in a cohort of patients receiving ACE inhibitor/angiotensin receptor blocker therapy, showed that hyperkalaemia associated risk when combined with spironolactone is much greater in real-life practice than observed in clinical trials. The risk of hyperkalaemia in heart failure patients was strongly associated with spironolactone use (odds ratio 